\title{
Estrategia de auditoria forense para la prevención de fraudes empresariales
}

\section{Forensic audit strategy for the prevention of business fraud}

\section{Estratégia de auditoria forense para a prevenção de fraudes comerciais}

\begin{abstract}
Manuel Rafael Quevedo-Barros I
mrquevedob@ucacue.edu.ec
\end{abstract}

Pablo Eduardo Barahona-Vásquez II

pbarahona@ucacue.edu.ec

Jorge Oswaldo Quevedo-Vázquez ${ }^{\text {III }}$

joquevedov@ucacue.edu.ec

\author{
Glenda Maricela Ramón-Poma IV \\ gramon@ucacue.edu.ec
}

Glenda Elizabeth Cabrera-Cárdenas V

glenda_cabrera81@hotmail.com

*Recibido: 22 de enero de 2019 *Aceptado: 29 de marzo de 2019 * Publicado: 05 de abril de 2019

I Magíster en Contabilidad y Auditoría, Contador Público, Licenciado en Administración. Docente de la Universidad Católica de Cuenca Sede Azogues, Cuenca, Ecuador.

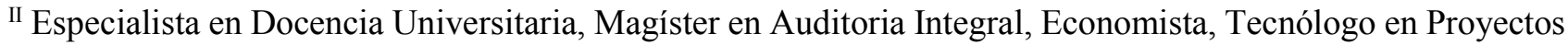
de Inversión. Docente de la Universidad Católica de Cuenca, Cuenca. Ecuador.

III Magíster en Administración de Negocios, Diploma Superior en Práctica Docente Universitaria, Economista, Licenciado en Economía y Finanzas, Subdirección de Posgrados Universidad Católica de Cuenca. Cuenca. Ecuador.

IV Especialista en Docencia Universitaria, Magíster en Dirección y Gestión de Empresas de Servicio, Ingeniera en Contabilidad y Auditoría (CPA), Docente de la Universidad Católica de Cuenca, Cuenca, Ecuador.

${ }^{\vee}$ Estudiante de la Universidad Católica de Cuenca, Cuenca. Ecuador. 


\section{Resumen}

El propósito del presente trabajo fue diseñar una estrategia de auditoría forense para la prevención de fraudes empresariales, que permita abarcar las metodologías y pasos de manera más sencilla y directa, a fin de contribuir con el buen manejo del dictamen sobre la incidencia de los estados contables como un aporte para los sistemas administrativos. Basado teóricamente en las concepciones que sobre auditoría forense y fraude empresarial exponen Rodríguez (2002) y Ciro (2017), respectivamente. Los materiales y métodos utilizados se inclinan hacia la investigación empírica (Martínez y Martínez: 1996), Cualitativa (Hernández, Fernández y Baptista: 2014), tipo Documental (Arias 2012), con un enfoque amplio y sistémico asentado en una configuración holística. Hurtado (2010). Las técnicas de recolección de información fueron el diseño bibliográfico de campo, la lectura general de textos, la observación (metanálisis) documental y el registro, el análisis de las fuentes, la revisión de la literatura especializada y la extracción de datos bibliográficos. Los instrumentos utilizados se ciñen al subrayado, el fichaje, las citas y notas de referencias, de ampliación de texto, la construcción y presentación de índices. De igual forma, la unidad de análisis está comprendida por el paradigma y la investigación misma. Las Técnicas de análisis se orientaron a la verificación bibliográfica de fuentes documentales, así como la lectura, interpretación y análisis en una profunda exploración epistémica. De lo encontrado en el proceso indagatorio de la investigación se recopiló una serie de información relevante para la construcción del diseño de la estrategia dirigida a la prevención de fraudes empresariales, de manera que se pueda caracterizar los aspectos fundamentales de los controles internos que afectan a los procesos contables.

Palabras Claves: Auditoria forense; fraudes empresariales.

\section{Abstract}

The purpose of this paper was to design a forensic audit strategy for the prevention of business fraud, which allows to cover the methodologies and steps in a simpler and direct way, in order to contribute to the proper handling of the opinion on the incidence of the financial statements. As a contribution for administrative systems. Based theoretically on the conceptions that on forensic audit and corporate fraud expose Rodríguez (2002) and Ciro (2017), respectively. The materials and methods used are inclined towards empirical research (Martínez and Martínez: 1996), Qualitative (Hernández, 
Fernández and Baptista: 2014), Documentary type (Arias 2012), with a broad and systemic approach based on a holistic configuration. Hurtado (2010). The information collection techniques were bibliographic field design, general reading of texts, documentary observation (meta-analysis) and registration, analysis of sources, review of specialized literature and extraction of bibliographic data. The instruments used are limited to underlining, signing, appointments and reference notes, text extension, construction and presentation of indexes. Similarly, the unit of analysis is comprised by the paradigm and the research itself. The analysis techniques were oriented to bibliographic verification of documentary sources, as well as reading, interpretation and analysis in a deep epistemic exploration. From what was found in the investigative investigation process, a series of information relevant to the construction of the strategy design aimed at the prevention of business fraud was compiled, so that the fundamental aspects of the internal controls affecting the companies could be characterized. accounting processes.

Keywords: Forensic Audit; business frauds

\section{Resumo}

O objetivo deste estudo foi o de projetar uma auditoria forense estratégia para a prevenção da fraude corporativa, que pode abranger metodologias e passos mais simples e maneira mais direta de contribuir para a boa gestão do parecer sobre o impacto das demonstrações financeiras como contribuição para os sistemas administrativos. Baseada teoricamente nas concepções que sobre auditoria forense e fraudes corporativas expõem Rodríguez (2002) e Ciro (2017), respectivamente. Os materiais e métodos utilizados inclinar-se para a pesquisa empírica (Martínez y Martínez: 1996) qualitativa (Hernández, Fernández e Baptista: 2014), tipo de Documentário (Arias 2012), com uma abordagem ampla e sistêmica baseada em um ambiente holístico. Hurtado (2010). As técnicas de coleta de informações foram desenho de campo bibliográfico, leitura geral de textos, observação documental (metanálise) e registro, análise de fontes, revisão de literatura especializada e extração de dados bibliográficos. Os instrumentos utilizados limitam-se ao sublinhado, assinatura, compromissos e notas de referência, extensão de texto, construção e apresentação de índices. Da mesma forma, a unidade de análise é composta pelo paradigma e pela própria pesquisa. As técnicas de análise foram orientadas para a verificação bibliográfica de fontes documentais, bem como para leitura, interpretação e análise em uma exploração epistêmica profunda. Que a encontrada na pesquisa 
Estrategia de auditoria forense para la prevención de fraudes empresariales

processo de investigação uma série de estratégia de construção relevantes, visando a prevenção de informações fraudes corporativas foram recolhidos, para que possa caracterizar os aspectos fundamentais de controles internos que afetam processos contábeis.

Palavras-chave: Auditoria forense; fraude comercial.

\section{Introducción}

En una economía sometida a constantes cambios y avances tecnológicos, de cara a la globalización, mantener el control y evitar los fraudes corporativos en las empresas se torna cada vez más complejo dado los avances tecnológicos actuales. Esta preocupación ha sido ampliamente expresada por las Naciones Unidas, el Banco mundial, la Organización de Estado Americanos, el Instituto Americano de la Administración Publica y el Instituto Latinoamericano contra la Corrupción.

En el mundo corporativo, son bien sabidos los escándalos de corrupción ocurridos desde la caída de la bolsa de valores de Nueva York en 1929; también, en el ámbito político se suscitó el caso Watergate en 1972, pasando por los más actuales acaecidos en las multinacionales: Parmalat (Italia), Enron (USA), World Com (USA), Royal Ahold (Holanda), Tyco (USA), Global Crossing (USA) y la Petrolera Yukos (Rusia), que tuvieron gran repercusión en la merma económica y su posterior quiebre; no obstante, en todos los niveles empresariales existen hechos como estos.

En ese contexto, se han organizado algunas delegaciones como la Comisión de Valores Mundial (IOSCO) y la organización internacional para el sector público (INTOSAI) que en 2005, emitieron los lineamientos para mejorar la praxis contable en las empresas, denominado "Fraud Report", brindando a los profesionales de la contabilidad homogeneidad en el uso de esta herramienta y dieron origen a una serie de regulaciones por parte de la SEC (Securities and Exchange Commission). Más tarde, en 2011, el Consejo de Normas Internacionales de Contabilidad (IASB) y el emisor de normas nacionales de los Estados Unidos, junto al Consejo de Normas de Contabilidad Financiera (FASB) publicaron un Memorando de Entendimiento para crear un conjunto común de normas contables globales de alta calidad.

Con ello, se pudo tener acceso a una serie de recomendaciones y pasos atenientes al fraude ocupacional y actos ilícitos a través del análisis de los estados financieros en los que se pueden detectar 
actividades ilegales como: falsificación de nómina o nominas paralelas de empleados, rendición de gastos inadecuados, falsificación de balances, hurto de dinero o bienes, derivados de la extorsión y corrupción empresarial. Sin embargo, de la empírea y el arqueo bibliográfico realizado por los autores subyace la necesidad de diseñar una estrategia de auditoria forense para la prevención de fraudes empresariales, que permita abarcar las metodologías y pasos de manera más sencilla y directa, a fin de contribuir con el buen manejo del dictamen sobre la incidencia de los estados contables como un aporte para los sistemas administrativos.

\section{Materiales y Métodos}

\section{Breve reseña del origen de la auditoría contable y su objeto de estudio.}

Según Maiola (2014:17), el origen de la auditoría contable "se remonta al año 254 A.C. con la civilización sumeria, como es descrito en los papiros de Zenón”; por su parte, (Chapman, 1952), cree que sus antecedentes "se encuentran más lejanos en la actividad desarrollada por los Escribas del antiguo Egipto". En opinión de (Bet 2015:64), para algunos tratadistas "data desde el advenimiento de la actividad comercial en la Inglaterra del siglo XIII con la a expansión empresarial y la Revolución Industrial"

Lo cierto es, que ocurren grandes transformaciones socioeconómicas desde la segunda mitad del siglo XVIII e inicios del siglo XIX con la liberación de los mercados hacia el comercio internacional a partir de los Tratados de Utrecht específicamente en el año 1713, cuyo proceso potencia las relaciones monetario-mercantiles entre las naciones Europeas y su extensión hacia las colonias; lo que posibilitó el nacimiento de la contaduría pública como profesión en el ambiente empresarial, conocido actualmente como auditor, "producto del acrecentamiento de los negocios, así como el movimiento de grande sumas de capital que pasan de ser capitales individuales a conformar las llamadas sociedades anónimas”. (Rodríguez, Martínez y Cudeiro; 2012).

La referencia más fidedigna que se tiene de los detectives contables data de 1824 en la ciudad de Glasgow, Escocia donde un profesional ofrece su testimonio y trabaja de experto evaluador como servicio de peritaje en tribunales y consejos, tomando su auge hacia los años 1900 en Estados Unidos e Inglaterra. Para abordar los delitos de evasión fiscal, son creados los agentes especiales contables por el IRS americano denominados investigadores forenses, a quienes se les atribuye como mayor 
Estrategia de auditoria forense para la prevención de fraudes empresariales

logro la encarcelación de AL Capone. Durante la segunda guerra mundial, la brigada de investigación criminal del FBI empleó a más de 500 auditores y contadores como agentes, examinado toda transacción financiera, pero no es sino hasta 1946 cuando se publica "La Contabilidad Forense: Su lugar en la Economía de Hoy, escrito por Maurice E. Peloubet en New York. (Rodríguez 2002:5).

Auditar implica "someter a un proceso de revisión, por un experto profesional suficientemente cualificado, determinado procedimiento, actividad, informe, proceso, entre otros, con intención de obtener un alto grado de garantía de la correcta elaboración o desarrollo de los mismos", así los expresa Biler (2017:2); en otras palabras, es una auditoria especializada que se enfoca en indagar, divulgar y atestar delitos en el desarrollo de las funciones operativas del cargo. Es, "la ciencia que permite reunir y presentar información financiera, contable, legal, administrativa e impositiva, para que sea aceptada por una corte o un juez en contra de los perpetradores de un crimen económico" Cano (2004:6), dado que conlleva un procedimiento para tal fin dentro de la investigación.

En palabras (Rodríguez 2002:21), la auditoría forense debe entenderse como “el proceso de recopilar, evaluar y acumular evidencia con la aplicación de normas, procedimientos y técnicas de auditoría, finanzas y contabilidad, para la investigación de ciertos delitos, que se pueden agrupar como fraudes, corrupción y terrorismo". De la revisión de la literatura se puede referir, que el término forense es una expresión moderna que infiere la ubicación, recolección y posterior presentación de evidencias contundentes para la aplicación de la ley con el uso de los principios de la ciencia y la tecnología que se pueden aplicar para identificar, recuperar, reconstruir o analizar las pruebas de un delito durante una investigación de carácter civil o penal y ser dirimida en los tribunales tal y como lo afirman Díaz y Pérez (2015:45), “permite reunir y presentar información”, como parte de sus tareas.

Para Estupiñán (2006), el concepto de fraude es referido de manera inicial como el "acto intencional que puede involucrar: la manipulación o falsificación de documentos, el uso indebido de recursos, la supresión u omisión de los efectos de las transacciones en los riesgos y la aplicación indebida de las políticas de contabilidad". Este hecho, es calificado como un delito ético, moral y económico, ya que se realiza para beneficio propio y perjudica a un tercero.

Al reseñar el fraude empresarial, Ciro (2017:1), lo describe como una "acción encaminada a eludir cualquier disposición legal, ya sea esta fiscal, penal o civil, siempre que con ello se produzca perjuicio 
Estrategia de auditoria forense para la prevención de fraudes empresariales

contra el Estado o en contra de un patrimonio". Es decir, tiene incidencia penal jurídicamente hablando y es castigado por las leyes y códigos al ser comprobado el timo o estafa.

De allí que los auditores externos según explica Wesberry (2004), deben familiarizarse con las dos formas que se manifiestan en el crimen económico. Los fraudes empresariales tal y como lo señala el autor son del tipo Corporativo y Ocupacional o Laboral conforman parte del objeto de estudio de la auditoría forense.

El crimen de tipo corporativo u ocupacional es cometido por la empresa (alta gerencia para perjudicar a los usuarios de estados financieros, tales como prestamistas, inversionistas, accionistas, estado sociedad) y el delito de tipo laboral, son aquellos delitos perpetrados por empleados en el curso de sus ocupaciones contra sus empleadores como malversación de activos, por ejemplo.

En atención a la naturaleza y el contenido del problema que atañe a la auditoría contable, y de acuerdo con el propósito formulado, se asume que la estrategia metodológica es empírica, a partir de la premisa de que "solo la experiencia garantiza un conocimiento verdadero" (Martínez y Martínez 1996), dado que al observar y vivir la realidad a través de la práctica rutinaria se obtiene un discernimiento e idea certera de los hechos.

Asimismo, esta investigación se ubicó en el contexto del paradigma cualitativo que para Hernández, Fernández y Baptista (2014: 36): “se enfoca a comprender y profundizar los fenómenos, explorándolos desde la perspectiva de los participantes en un ambiente natural y en relación con el contexto" es por tanto necesario, referir la intencionalidad de escudriñamiento acerca de los fraudes empresariales y su prevención mediante la auditoria forense. La investigación se orientó prioritariamente hacia esta modalidad por los requerimientos específicos del tema que se estudió, donde se debió captar la reflexión intrínseca de los investigadores en el proceso, así como las interpretaciones que permitió aprovechar una visión más global y aproximada de la realidad estudiada.

El tipo de investigación es documental, que según Arias (2012:15), es "el estudio de problemas con el propósito de ampliar y profundizar el conocimiento de su naturaleza", en este sentido, este tipo de diseño operacional se ciñó a una alineación expositiva, puesto que sustentó las interpretaciones necesarias a partir de teóricos que fundamentaron las capacidades analíticas, interpretativas de los investigadores, recurriendo a documentos que propugnaron las elucubraciones con un enfoque amplio 
y sistémico asentado en una configuración holística, como bien lo explica Hurtado (2010:99), un "proceso dinámico que permite explorar, describir, analizar, comparar, explicar, predecir, proponer, modificar, confirmar y evaluar la búsqueda del conocimiento en múltiples abordajes", o lo que es lo mismo, desde la complementariedad, que tribute hacia la integración de elementos que pudieran aportar una solución al fenómeno observado.

Las técnicas de recolección de información fueron el diseño bibliográfico de campo en la cual se definen las categorías en estudio; la lectura general de textos, la observación (metanálisis) documental y el registro, el análisis de las fuentes, la revisión de la literatura especializada y la extracción de datos bibliográficos. Los instrumentos utilizados se ciñen al subrayado, el fichaje, las citas y notas de referencias, de ampliación de texto, la construcción y presentación de índices. De igual forma, la unidad de análisis está comprendida por el paradigma y la investigación misma, ya que el primero incide en el otro, por referirse a un contexto y objeto de estudio específico.

Las Técnicas de análisis se orientaron a la verificación bibliográfica de fuentes documentales, así como la lectura, interpretación y análisis en una profunda exploración epistémica que permitió examinar los conceptos claves y sustentar las ideas sobre los argumentos teóricos, que ayudan a entender mejor las categorías relevantes, de tal manera que las interpretaciones se derivaron de la próxima aplicación de estrategias analíticas sobre los tópicos indagados.

\section{Hallazgos}

De lo encontrado en el proceso indagatorio de la investigación se recopiló una serie de información relevante para la construcción del diseño de la estrategia dirigida a la prevención de fraudes empresariales, de manera que se pueda caracterizar los aspectos fundamentales de los controles internos que afectan a los procesos contables y entregar información útil para la gerencia y los directores además del orientar el servicio prestado hacia el cumplimiento de leyes tributarias, previsional, normas éticas, responsabilidad social empresarial y sustentabilidad, dada la vulnerabilidad en la que se encuentran con respecto a este flagelo económico y moral.

Para nadie es un secreto que estas actividades ilícitas son catalogados como crímenes y están altamente penados en la jurisprudencia, en virtud de las grandes pérdidas incalculables que se generan, evitando así la oportunas de crecimiento para la empresa, una merma significativa en las inversiones y el 
estancamiento, bancarrota o lo que es lo mismo, pérdida definitiva del negocio y, por ende pérdida de empleos.

En ese tenor, se ha observado que la percepción de los autores es que, por lo general, son los altos gerentes y funcionarios con cargos de más responsabilidad quienes, en su mayoría, cometen este tipo de actos, reforzando así la tesis del triángulo del fraude originada a partir de la hipótesis de Cressey (1973:30), aún vigente:

"Las personas de confianza se convierten en violadores de confianza" cuando se conciben a sí mismos teniendo un problema financiero que no es compartible, son conscientes de que este problema puede ser resuelto en secreto violando la posición de confianza financiera que tienen, y son capaces de aplicar a su propia conducta en esa situación, verbalizaciones que les permitan ajustar sus concepciones de sí mismos como personas de confianza, con sus concepciones de sí mismos como usuarios de los fondos o propiedades encomendadas".

El concepto del triángulo del fraude acuñado por Cressey (op.cit), refiere que los individuos que realizan acciones delictivas en relación a estafas y fraudes financieros que involucran a las empresas son trabajadores de la misma o por lo menos tienen un cómplice que funge como enlace de sus fechorías cuya motivación principal es el enriquecimiento ilícito para su propio beneficio, en el que por el manejo y poder que tienen ven la oportunidad sin la más mínima racionalización.

En tal sentido, los autores presentan a continuación el diseño estratégico de auditoria forense para la prevención de fraudes empresariales, el cual trata de una propuesta compuesta por una metodología macro dimensionada en tres partes: La primera es la denominada "Fases", la segunda designada como "Etapas" la tercera "Contenidos". En razón a las Fases esta comprende sus "Etapas" de acuerdo a la fase que a su vez están sujetas a un "Contenido". La estructura secuencial de las Fases son: Planificación, Ejecución, Información y Monitoreo. (Ver Cuadro Nro. 1) 
Dom. Cien., ISSN: 2477-8818

Vol. 5, núm. 2, abril 2019, pp. 402-415

Estrategia de auditoria forense para la prevención de fraudes empresariales

Cuadro 1. Estrategia de Auditoria para la Prevención de Fraudes Empresariales

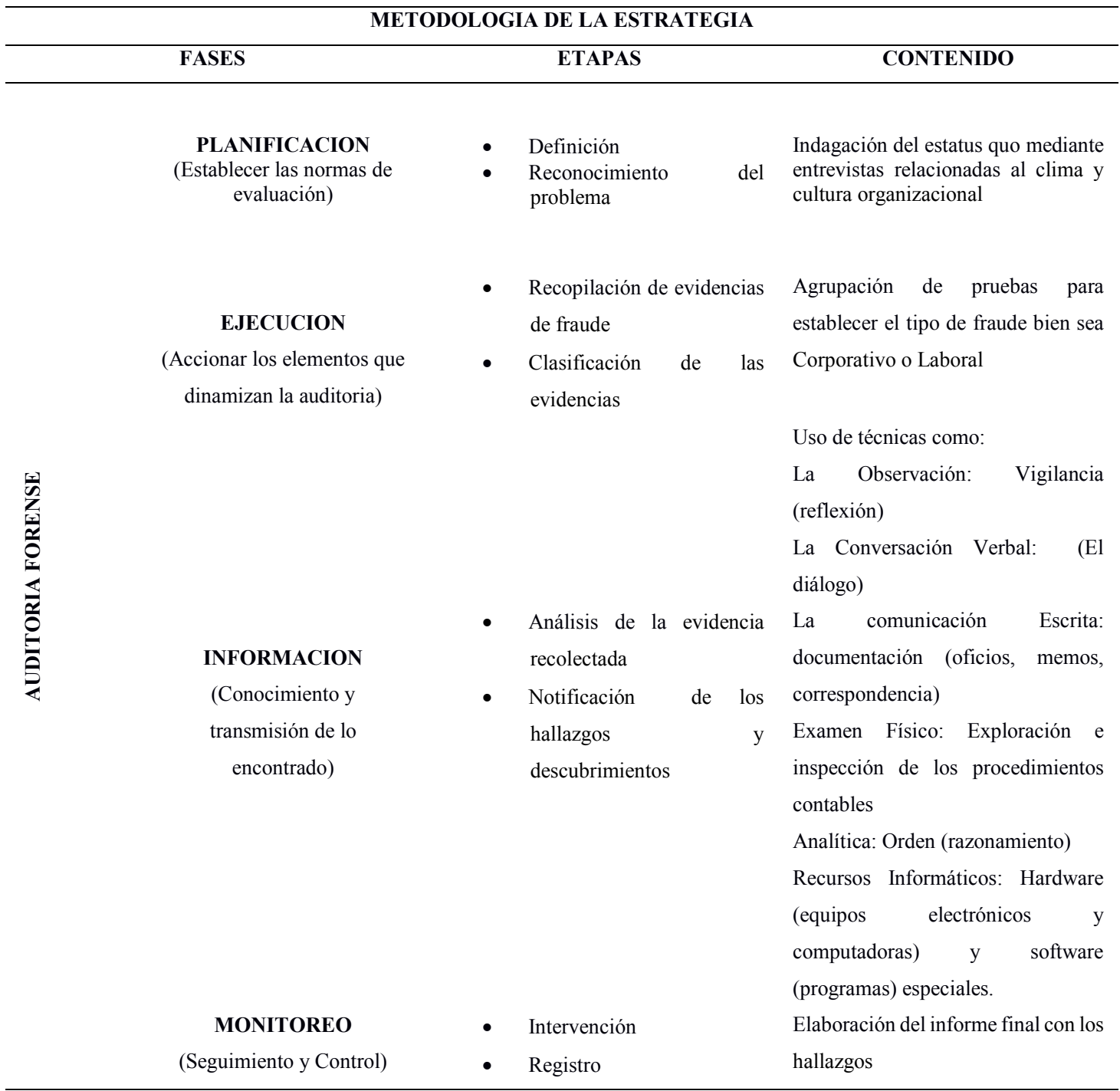

PREVENCION DE FRAUDES EMPRESARIALES

Fuente: Revisión Bibliográfica de autores (2019). Elaboración propia

En La primera fase de la estrategia denominada "Planificación", se establecen las normas de evaluación y se proyecta la programación del tiempo, el espacio y los recursos con los que se cuenta, para luego llevar al plano real lo escrito en el papel de trabajo. Consiste en realizar un diagnóstico de la situación, que permita identificar las debilidades de los procesos claves, a manera de recoger la información sobre el hecho o situación observada, relativo a los sujetos que son objeto de la investigación; el cual se alzará con la intención de utilizarlo en los pasos subsiguientes como marco de referencia, a través de las etapas de Definición y Reconocimiento del Problema, cuyo contenido 
comprende la Indagación del estatus quo, mediante entrevistas relacionadas al clima y cultura organizacional, como base para determinar algunos aspectos inherentes a la empresa.

En la fase de "Ejecución", se accionan los elementos que dinamizan la auditoría en dos etapas: Recopilación de evidencias de fraude para compilar los elementos requeridos, en caso de ser necesario y la Clasificación de las evidencias para conocer el tipo de fraude, los cuales pueden ser Corporativos o Laborales y establecer así los parámetros operativos que gestionen los aspectos fundamentales de la auditoría.

La siguiente fase es la de "Información". Aquí se desarrolla el conocimiento y posterior transmisión de lo encontrado analizando en un primer momento la evidencia recolectada para hacer la notificación de los hallazgos y descubrimientos que corresponden en el proceso a través del uso de técnicas como: la Observación como primera instancia de la vigilancia reflexiva; la Conversación Verbal mediante el diálogo e intercambio de opiniones; la comunicación escrita, donde se revisa exhaustivamente los documentos tales como oficios, memos y correspondencia en general ya sea física o digital; el Examen Físico, momento en el cual se explora e inspeccionan los procedimientos contables; la Analítica, que es orden sistémico y razonamiento de acuerdo a los estándares y finalmente los Recursos Informáticos, como el hardware (equipos electrónicos y computadoras) y software (programas) especiales y adecuados para tal fin.

Por último, se encuentra la fase de Monitoreo, que permite la intervención propiamente dicha para la prevención de fraudes, como parte de la reestructuración necesaria, en aras de evitar daños colaterales; la misma es acompañada de un despliegue en la sistematización del registro de los hechos y pruebas para finalmente, elaborar el informe definitivo con sus hallazgos.

En relación con los contenidos de la estrategia aquí propuesta, se describe en esta parte la significación de cada uno de las unidades que la constituyen:

\section{Objetivo General}

Proponer una estructura organizativa para la operatividad de la auditoría forense a fin de prevenir fraudes empresariales. 


\section{Objetivos Específicos}

- Incidir en políticas, programas y acciones que involucren, lo organizativo, lo político y lo administrativo.

- Describir una metodología de trabajo eficiente.

- Orientar la práctica sistemática y metodológica en los auditores forenses.

\section{Características}

- Fortalecer el proceso que se lleva a cabo en las auditorías forenses dentro de las empresas.

- Reforzar los lineamientos internacionales

- Identificar los problemas para la posterior reestructuración de los procedimientos administrativos y contables

- Desarrollar un sistema de innovador que renueve los conocimientos, actitudes y habilidades de los auditores forenses.

- Coadyuvar en el desarrollo integral de los actores involucrados en el proceso de auditoria forense para optimizar la operatividad de misma.

\section{Reflexión Final}

En primer lugar, es importante advertir que el fraude financiero, por su propia naturaleza viene de los más altos niveles de dirección y responsabilidad de las empresas, derivado de conductas deshonestas de sus ejecutivos, manifestándose así mismo en todas las estructuras desde las pequeñas y medianas empresas hasta las grandes multinacionales, dada la interrelaciona con otros dos sectores tanto públicos como privados y la sociedad civil en general compuesta por clientes y usuarios, en la que se distinguen distintos credos y valores ético morales no acordes con sus ambiciones.

En ese contexto, todo tipo de empresa agropecuaria, minera, industrial, comercial y de servicios es susceptible a fraudes financieros y su vulnerabilidad persiste en todos los niveles y no se pueden evitar definitivamente pero si se pueden crear mecanismos que permitan llevar un mejor, riguroso y estricto control con políticas antifraudes que se implanten las empresas como por ejemplo la estrategia que aquí se diseñó y así prevenir el crecimiento de las modalidades delictivas; el fraude financiero y la apropiación indebida de activos empresariales. 


\section{Referencias Bibliográficas}

Arias (2012). El proyecto de investigación. 6ta edición. Editorial Episteme, Venezuela

Bet, L. (2015). La auditoría como ejercicio generador de herramientas gerenciales para reducir los riesgos de fraude en los entes económicos SABER, CIENCIA Y Libertad ISSN: 1794-7154 Vol. 10, No.2

Biler-Reyes, S. (2017). Auditoria. Elementos esenciales. Dominio de las Ciencias, 3(1), 138-151.

Cano \& Lugo. (2004). Auditoría Forense: en la investigación criminal del lavado de dinero y activos.Ediciones ECOE.- 2da. Edición, Colombia, pág. 2.

Chapman, W. (1952). "Responsabilidad del auditor externo", Revista de Ciencias Económicas Nº 35, Marzo-Junio.

Ciro, C. (2017). Fraude Empresarial. Universidad Militar Nueva Granada. Facultad de Relaciones Internacionales Seguridad y Defensa. Ensayo. Colombia. Disponible en https://repository.unimilitar.edu.co/bitstream/handle/10654/16667/CesarAug;jsessionid=1FB 723410F9CC645C8A491350B573083? sequence=1. Consultado el 02/06/2019.

Cressey, D. (1973). Dinero de los demás. Montclair: Patterson Smith.

Díaz y Pérez (2015). La auditoría forense: Metodología y herramientas aplicadas en la detección de delitos económicos en el sector empresarial de la ciudad de Cartagena.

Estupiñan, R. (2006). Control interno y fraudes con base en los Ciclos transaccionales: Análisis de informe COSO I y II. Bogotá: Eco Ediciones Ltda.

Hernández, R., Fernández, C. y Baptista, P. (2014). Metodología de la Investigación. 6ta Edición. Editorial Mc Graw Hil education.

Hurtado de Barrera, J. (2010). Guía para la comprensión holística. Tercera Edición, Fundación Sypal: Caracas. (Parte II Capítulo 3 y 4). 
Estrategia de auditoria forense para la prevención de fraudes empresariales

Lozano, N. (2013). Diagnóstico de gobierno corporativo como mecanismo en la prevención Maiola, O. (2014). El fraude y los controles en los sistemas contables. Contabilidad y auditoría, (38), 51.

Martínez, L. y Martínez, Hugo, (1996). Diccionario de Filosofía. Panamericana Editorial. Bogotá, Colombia.

Rodríguez, N. C., Martínez, N. R. D., \& Cudeiro, Y. A. (2012). La auditoría como una disciplina dentro de las ciencias contables. Enfoques teóricos y metodológicos de su praxis. Observatorio de la Economía Latinoamericana, (166).

Wesberry, J. (2004): La Auditoría Interna en su Combate a la Corrupción y el Fraude. Cuentas Claras. No. 8, pp. 1-8. 